\title{
EXPERIENCIA UNIVERSITARIA SOBRE LAS FUNCIONES DEL EDUCADOR/A SOCIAL CON TECNOLOGIAS 2.0.
}

\section{UNIVERSITY EXPERIENCE ON THE FUNCTIONS OF SOCIAL EDUCATOR WITH 2.0 TECHNOLOGIES.}

\author{
Eloy López Meneses; eloy3000@gmail.com \\ Vicente J. Llorent García; vjllorent@uco.es \\ Esther Fernández Márquez; estfdez@gmail.com
}

Universidad Pablo de Olavide

\section{RESUMEN}

En el artículo se describe una experiencia de innovación en enseñanza universitaria, con presentaciones educativas interactivas sobre las principales funciones del Educador/a Social.

Entre sus objetivos destacan: Reflexionar sobre las principales funciones del profesional de la Educación Social, favorecer el rol activo y autónomo de los estudiantes en el proceso de construcción del conocimiento. La experiencia educativa ha corroborado que las principales funciones reconocidas para este alumnado son orientar (66,15\%), educar y enseñar conocimientos y experiencias $(52,31 \%)$, motivar $(41,54 \%)$, dinamizar $(40 \%)$, informar y promover $(27,69 \%)$, tutorizar $(24,62 \%)$, evaluar $(18,46 \%)$, investigar $(16,92 \%)$, intervenir socialmente y favorecer los procesos de mediación $(13,85 \%)$.

PALABRAS CLAVE: Educación superior, aprendizaje en red, estrategia de enseñanza virtual, web 2.0., innovación educativa.

\section{ABSTRACT}

The article describes an innovative experience in university teaching, with interactive educational presentations on key functions of Social Educator.

Its objectives include: Reflect on the main professional functions of social education, promote active and independent role of students in the process of building knowledge, promote learning. The study confirmed that the main functions for students are: guide $(66.15 \%)$, educate, and teach or transfer knowledge and experience $(52.31 \%)$, motivate $(41.54 \%)$, animate and stimulate $(40 \%)$, inform and promote $(27.69 \%)$, facilitate processes and tutoring $(24.62 \%)$, evaluate $(18.46 \%)$, investigate $(16.92 \%)$, be socially involved and encourage mediation processes $(13,85 \%)$.

KEYWORDS: Higher education, online learning, e-learning strategy, web 2.0., Educational innovation. 


\section{INTRODUCCIÓN}

La pujanza de la sociedad de las tecnologías y de la información, y la irrupción del fenómeno de la web 2.0 en los contextos formativos universitarios han provocado un profundo viraje en las funciones que deben desempeñar los docentes. La capacitación didáctica y tecnológica del profesorado se está convirtiendo en un imperativo para hacer frente a las nuevas situaciones de enseñanza-aprendizaje desarrolladas en escenarios virtuales y/o con el apoyo de herramientas tecnológicas. (Del Moral y Villalustre, 2012).

En este sentido, uno de los grandes retos a los que se enfrenta la Educación es, sin duda alguna, el uso de las nuevas Tecnologías de la Información y la Comunicación (TIC), las cuales merecen concebirse como herramientas tecno-didácticas para el fortalecimiento del docente y para la significación y re-significación de los aprendizajes cotidianos que adquieren los educandos en los contextos educativos (Soto, 2010). Dentro de las TIC se encuadra la llamada web 2.0, es decir, el uso de Internet de forma bidireccional, compartiendo información, creando contenidos de forma conjunta, etc. (Montero, 2010). La utilización de ésta en la Educación Superior supone nuevos retos e implicaciones pedagógicas para los procesos educativos tanto presenciales como híbridos y virtuales (Suárez y López Meneses, 2011).

El incremento exponencial de aplicaciones basadas en la web se está consolidando como un medio importante en el ámbito educativo (Pulichino, 2006; Saeed, Yang y Sinnappan, 2009; Aguaded, Guzmán y Tirado, 2010). De hecho, para la formación del profesorado universitario se ha convertido en factor clave en las experiencias universitarias de innovación pedagógica, en el contexto de los nuevos retos del Espacio Europeo de Educación Superior (Aguaded y López Meneses, 2009). La Enseñanza Universitaria se ha fundamentado en un modelo metodológico centrado en el docente, con énfasis en la transmisión de contenidos y su reproducción por parte del estudiantado, la lección magistral y el trabajo individual. Sin embargo, enseñar a través de las TIC, demanda una serie de cambios que generan una ruptura de este modelo, al mismo tiempo que pueden suponer un avance hacia la calidad de la Educación Universitaria (Aguaded, López Meneses y Alonso (2010 a y b).

De igual manera, los docentes o inmigrantes digitales (Prensky, 2004), con la aparición de entornos interactivos 2.0 más abiertos, colaborativos y gratuitos, pueden utilizarlos como recursos didácticos para la implementación de metodologías más flexibles, activas y participativas en coherencia con la convergencia europea. Asimismo, los inmigrantes digitales deben utilizar en menor medida las metodologías centradas en el profesor (expositivas y pasivas) para ir evolucionando hacia otras metodologías donde el estudiante sea el protagonista (activas, dinámicas y participativas) (Miranda, Guerra, Fabbri y López Meneses, 2010).

\section{ESCENARIO DE LA INNOVACIÓN DIDÁCTICA}

Diferentes estudios realizados sobre el aprendizaje en red están poniendo claramente de manifiesto que la modalidad de formación virtual, es una cuestión notablemente diferente del simple hecho de utilizar una plataforma como repositorio de diferentes objetos de aprendizaje (Valverde y López Meneses, 2009). 
En concreto, nuestra investigación parte de una experiencia universitaria sobre las principales funciones del educador/a social desarrollada durante el curso académico 20112012 con estudiantes que cursaban la asignatura de «Tecnologías de la Información y la Comunicación en Educación Social», perteneciente al primer curso de la titulaciones de Grado de Educación Social y Doble Grado en Educación y Trabajo Social que se imparte en la Facultad de Ciencias Sociales de la Universidad Pablo de Olavide.

El presente estudio emana del proyecto: "Formación didáctica en Cloud Computing: Competencias digitales, estrategias didácticas y e-actividades con tecnología Web 2.0 en el EEES", desarrollado en el marco de los Proyectos de Innovación y Desarrollo Docente de la Universidad Pablo de Olavide (UPO), financiado por el Vicerrectorado de Docencia y Convergencia Europea de dicha Universidad. En el artículo se describe una experiencia de innovación en enseñanza universitaria, con presentaciones educativas interactivas sobre las principales funciones del Educador/a Social.

Respecto a otras experiencias virtuales universitarias, el lector interesado puede consultar los trabajos de Cabero, López Meneses y Ballesteros (2009), López Meneses y Llorente (2010), Aguaded, J. I., López Meneses, E y Alonso, L. (2010b) o López Meneses, Domínguez, Álvarez y Jaén (2011). A continuación, se muestran las intenciones didácticas, el proceso y los resultados más relevantes obtenidos durante el desarrollo de esta intervención.

\section{OBJETIVOS DE LA EXPERIENCIA.}

Como objetivo general de la experiencia señalar que se atiende a comprender las percepciones que los nuevos estudiantes de los Grado en Educación Social y Trabajo Social, poseen acerca de la figura y el rol docente, implícito al ámbito profesional en el que desarrollarán sus futuros trabajos, y contrastar estas concepciones con las aportaciones realizadas por diferentes autores.

Este objetivo, se concreta a nivel educativo, en los siguientes de carácter más específico:

- Fomentar el papel activo y autónomo del estudiantado en el proceso de construcción del conocimiento.

- Reflexionar sobre las principales funciones del educador/a social y el trabajador/a social.

- Favorecer el aprendizaje multimodal entre los y las estudiantes.

- Diseñar y elaborar presentaciones multimedia interactivas con aplicaciones relacionadas con el software social (Prezi).

- Fomentar el andamiaje socio-cognitivo.

- Promover la creatividad digital a través de recursos multimedia 2.0.

\section{METODOLOGÍA DEL ESTUDIO.}

La metodología desarrollada fue de corte cualitativo y descriptivo, siendo el propio evaluador el encargado de extraer, elaborar e interpretar los resultados a través de un proceso cíclico, simultáneo e interactivo. La muestra estuvo formada inicialmente por los/as 
estudiantes de las dos titulaciones, pero al ser una experiencia optativa sólo la realizaron 65 estudiantes.

Para el análisis cualitativo de las diapositivas informatizadas recibidas al correo electrónico del profesor $y$, posteriormente, implementadas en el espacio virtual: http://practica4000.jimdo.com/ se revisaron las presentaciones interactivas analizando las palabras y los conjuntos visuales de significados como unidades de registro. Posteriormente se transcribió y categorizó la trama visual-conceptual tomando como marco de referencia las pautas establecidas por diferentes autores (Bogdan y Biklen, 1992; Miles y Huberman, 1994):

- Fase Primera: Reducción de datos. Constituye la realización de procedimientos racionales que consisten en la categorización y codificación de los datos, identificando y diferenciando unidades de significado. Los procedimientos son:

Categorización de los datos. La categorización implica la simplificación y selección de información para hacerla más manejable. Este proceso implica varias subfases:

- Separación de unidades. Consiste en separar segmentos de información siguiendo algún tipo de criterio como puede ser espacial, temporal, temático, gramatical.

- Identificación y clasificación de unidades. Consiste en clasificar conceptualmente las unidades que son cubiertas por un mismo tópico con significado. El procedimiento puede ser inductivo, es decir, a medida que se van examinando los datos, o deductivo, habiendo establecido previamente el sistema de categorías sobre el que se va a categorizar, tras la revisión de literatura específica sobre la temática objeto de estudio. Normalmente esta clasificación suele ser mixta.

- Síntesis y agrupamiento. Esta fase está unida realmente a la anterior dado que la propia categorización implica la síntesis. También está presente una vez que ha concluido el proceso de categorización y algunas categorías se agrupan en metacategorías.

Codificación. Es realmente la operación concreta y manipulativa por la que se asigna cada categoría a cada unidad textual. En este sentido, cada unidad seleccionada ha sido codificada para su recuento frecuencial.

- Fase segunda: Interpretación e inferencia. Por último, el proceso de análisis de datos se completó con una etapa en la que se procedió a la interpretación de las diferentes unidades de información categorizadas, ordenando de modo sistemático en tablas y representaciones gráficas la información obtenida para facilitar la fase de interpretación y explicación de los resultados.

En el siguiente apartado se muestra el desarrollo de la práctica universitaria virtual, los resultados y conclusiones. 


\section{DESARROLLO DE LA EXPERIENCIA UNIVERSITARIA}

La experiencia de innovación se desarrolló en la asignatura denominada "Tecnologías de la Información y la Comunicación en Educación Social», correspondiente al primer curso de la titulación de Grado de Educación Social y doble Grado en Educación Social y Trabajo Social, de la Universidad Pablo de Olavide, durante el curso académico 2011/ 12. Consta de doce sesiones de prácticas donde se enseña el diseño, desarrollo e implementación de diferentes utilidades, aplicaciones y recursos 2.0 (wikis, blogs, sindicación de noticias, foros de debates, Word clouds, elaboración de mapas conceptuales en línea, presentaciones educativas en red, implementación de redes sociales y profesionales...) de gran interés para el educador/a y el trabajador/a social. Algunas de éstas se pueden consultar en el siguiente repositorio: http://alacenadigital.blogspot.com/ (figura 1).

\section{Mochila digital}

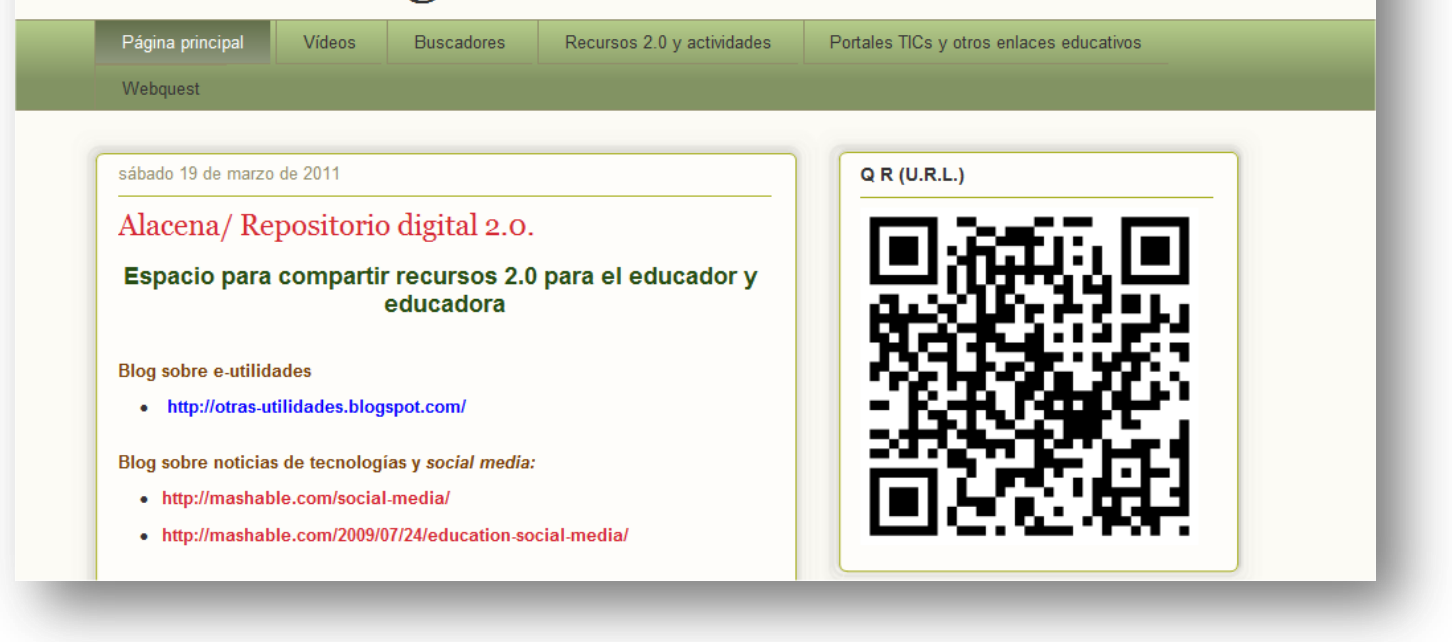

Figura 1. Blog sobre recursos 2.0 para la comunidad universitaria.

[http://alacenadigital.blogspot.com/].

Para que cada estudiante participara activamente en la construcción de su nuevo conocimiento -uno de los objetivos marcados en el proyecto- se pretendía que los futuros profesionales de la Educación Social y del Trabajo Social analizaran y reflexionaran sobre las funciones del profesional de la Educación Social y el Trabajador Social. Todo ello mediante la utilización de presentaciones multimedia educativas como recursos didácticos para su desarrollo académico y profesional.

La guía didáctica de las asignaturas de ambas titulaciones se articulaba alrededor de cuatro bloques temáticos sobre las TIC (esquema 1). En concreto, el estudio hace alusión al primer bloque temático (tema 3) que tiene como objeto de estudio el rol del educador en la Sociedad de la Información y el Conocimiento. 


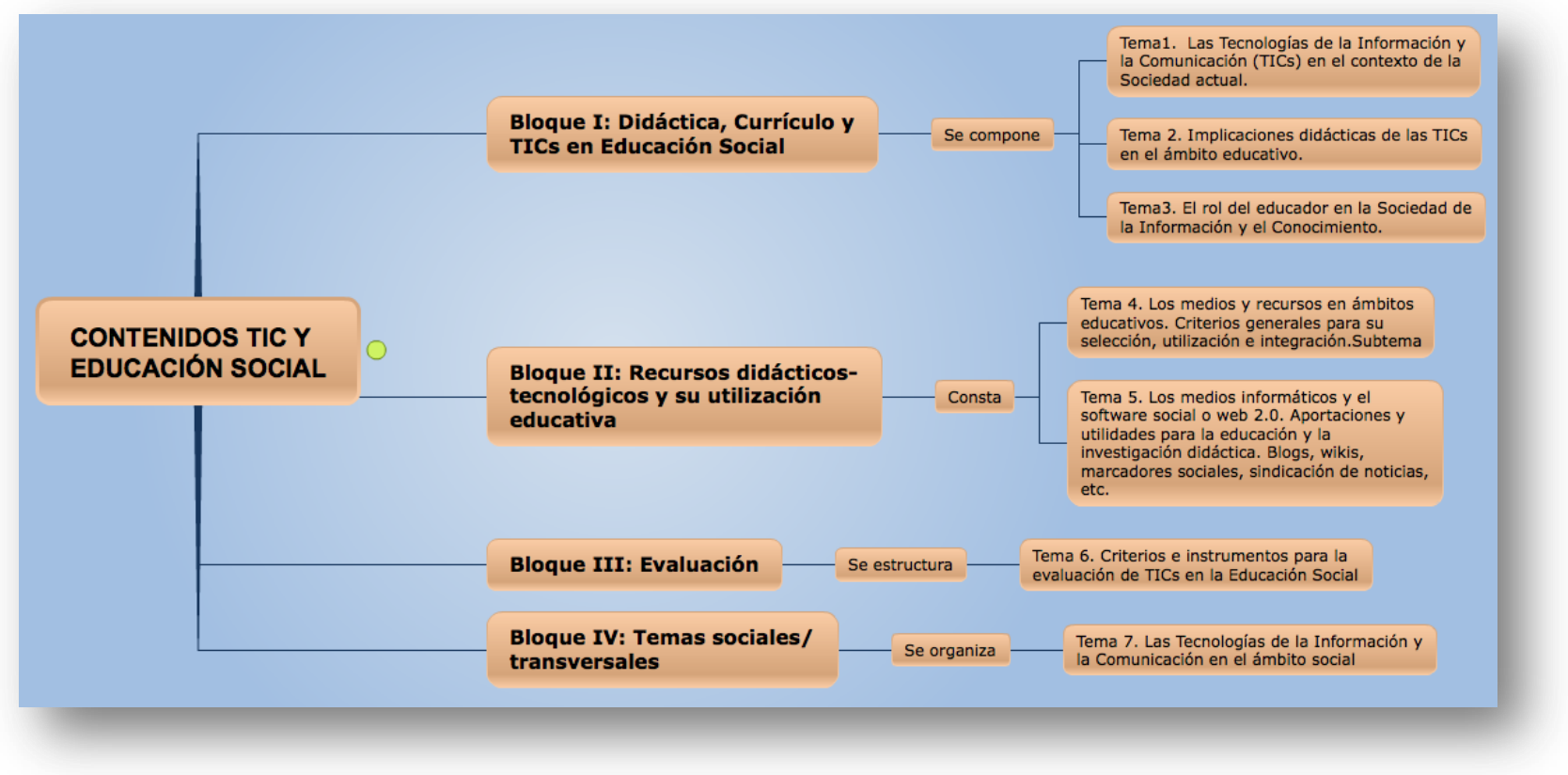

Esquema 1. Organigrama conceptual de los bloques de contenidos de la asignatura. [http://www.mindomo.com/view.htm?m=6cd2bf5f280e4e7bb7cca11b77b8beb1].

Para el desarrollo de la experiencia didáctica virtual durante el mes de marzo del curso académico 2011-12, en una primera instancia, se aconsejaba al alumnado que consultara el tema 3 denominado: "El rol del educador en la Sociedad de la Información y el conocimiento" y el artículo elaborado por el profesor Pere Marquès (2000) que estaba alojado en la plataforma WebCT-UPO de la Universidad Pablo de Olavide. Además, se sugería que el alumnado consultara en el portal bibliográfico de acceso libre (Dialnet): http://dialnet.unirioja.es/info/ayuda/qe, o bien, el servicio gratuito de consultas bibliográficas CiteUlike: http://www.citeulike.org/, como los diferentes materiales multimedia de apoyo educativo para profundizar en la temática, especialmente de la Biblioteca. Posteriormente, se dedicaba una sesión presencial teórica-participativa para la explicitación del objeto de estudio. Ésta se iniciaba con una actividad presencial de lluvias de ideas (brainstorming) con el estudiantado para la selección de los tres roles principales que debe tener un buen educador social de carácter individual, pasados unos 15 minutos, se realizaba un debate colectivo y se exponían en la pizarra tradicional los principales roles.

En la sesión de Enseñanza, Práctica y Desarrollo (EPD) que se realizaba en la aula de informática en grupos de 20 estudiantes, se familiarizaban con la aplicación de software social denominada Prezi (figura 2) para ello ser realizó el 26 de marzo de 2012, en concreto, en la séptima sesión práctica de la asignatura en el aula de informática de una hora y media una clase teórica-práctica para conocer y utilizar dicho recurso digital. 


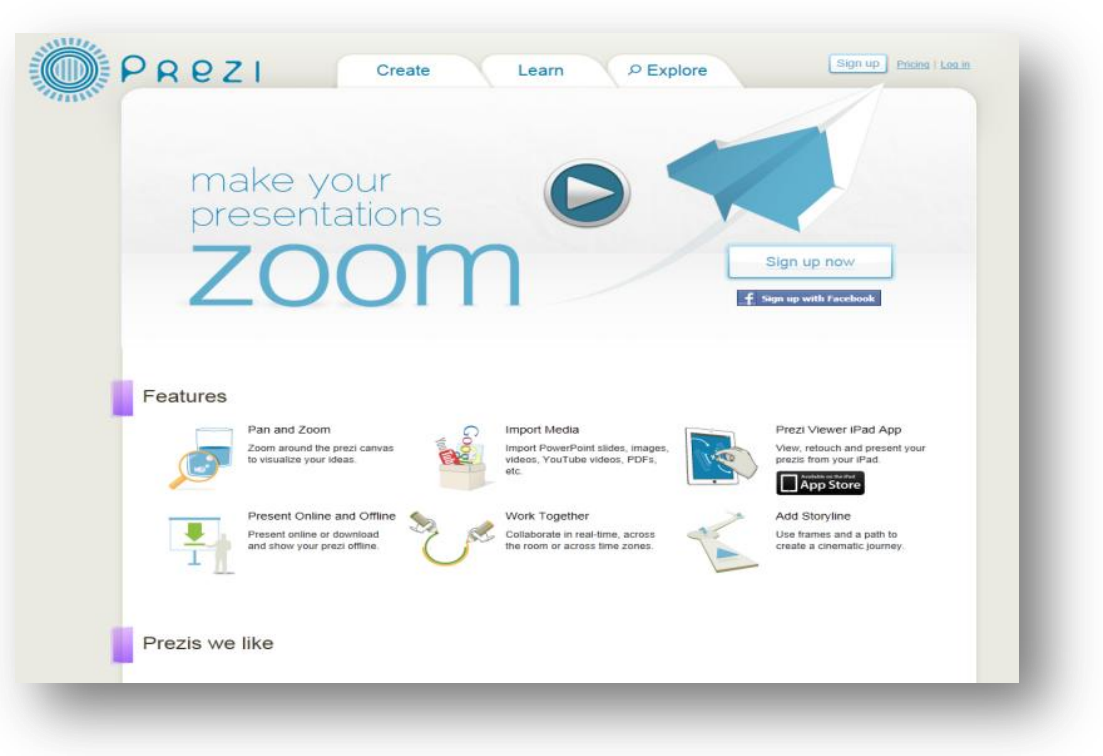

Figura 2. Software social PREZI.

\section{http://prezi.com/}

También es de resaltar que el software social PREZI, se encuentra posicionado en el puesto quinto por el Centro Learning \& Performance Technologies (C4LPT) (Hart, 2011).

Por otra parte, cada estudiante tenía libre elección para realizar la presentación de la forma más creativa posible, por lo que algunos/as estudiantes incluyeron imágenes, gráficas y vídeos educativos. Sirva como ejemplo el material interactivo realizado por una estudiante (figura 3).

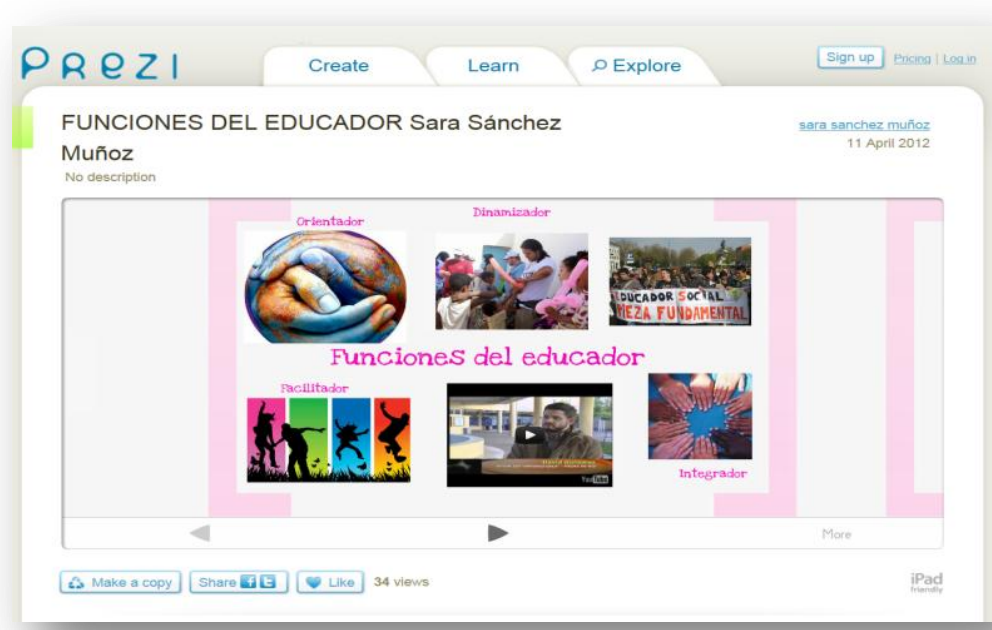

Figura 3. Prezi de una estudiante de la Titulación de Grado de Educación Social de la Universidad Pablo de Olavide de Sevilla. http://prezi.com/9syaz6mv-ua4/funciones-del-educador-sara-sanchez-munoz/

En referencia a la evaluación de la actividad era optativa con incremento de medio punto de la nota final de la asignatura. Seguidamente, se muestra (tabla 1) la matriz de valoración o rúbrica de la e-actividad. 


\begin{tabular}{|c|c|}
\hline CRITERIOS DE EVALUACIÓN & VALOR \\
\hline ENTORNO DIDÁCTICO & 5 PUNTOS \\
\hline $\begin{array}{l}\text { El mensaje didáctico multimedia está bien estructurado } \\
\text { destacando las principales funciones del educador/a } \\
\text { relacionado con las TIC. }\end{array}$ & 2 \\
\hline $\begin{array}{l}\text { Presentan algún esquema, diagrama, mapa conceptual } \\
\text { para facilitar la comprensión. }\end{array}$ & 1 \\
\hline $\begin{array}{l}\text { Cada diapositiva multimedia describe unas pocas } \\
\text { ideas con un predominio de la imagen. }\end{array}$ & 2 \\
\hline ENTORNO TÉCNICO & 3 PUNTOS \\
\hline $\begin{array}{l}\text { La presentación multimedia, en general, cumple el } \\
\text { criterio de simplicidad. }\end{array}$ & 1 \\
\hline El diseño es uniforme y su navegabilidad adecuada. & 1 \\
\hline $\begin{array}{l}\text { El uso de elementos multimedia (textos, imágenes, } \\
\text { gráficas, videos...) son legibles con colores y tipografia } \\
\text { adecuadas. }\end{array}$ & 1 \\
\hline OTROS ASPECTOS & 2 PUNTOS \\
\hline $\begin{array}{l}\text { Redacción clara, léxico adecuado y pertinente, sin } \\
\text { faltas de ortografia. }\end{array}$ & 1 \\
\hline La presentación es creativa e innovadora. & 1 \\
\hline
\end{tabular}

Tabla 1. E-rúbrica de la experiencia universitaria con la aplicación Prezi. U.R.L http://practica4000.jimdo.com/

\section{RESULTADOS DE LA EXPERIENCIA}

En primer lugar, cabe mencionar que a la hora de analizar el trabajo desarrollado por el alumnado no se estableció un sistema de categorías previas, sino que se siguió un proceso de categorización inductivo. Para ello, el análisis de datos se centró en aquellas palabras o conjuntos de significados que los y las estudiantes aportaban en su presentación multimedia digital y se consideraban como unidades de registro. A su vez, cada unidad seleccionada ha sido codificada con su recuento frecuencial. Seguidamente, se exponen los resultados obtenidos de la codificación del análisis de las presentaciones multimedia elaboradas por los/as estudiantes con relación a las funciones del educador/a y trabajador/a social. 
En la encuesta han participado un total de 65 alumnos y alumnas de 10 curso de Grado de la Especialidad de Educación Social, y doble Grado en Educación Social y Trabajo Social, en la Universidad Pablo de Olavide (Sevilla), a quienes se les ha solicitado que indicasen cuáles entienden ellos que son las principales funciones que forman parte de la figura del "Educador Social".

Señalando que el alumnado ha establecido una media de 5,11 funciones destacables a este profesional, y partiendo de que no existía ninguna tabla prestablecida de la cual tomar las posibles respuestas, reseñar que de entre los resultados obtenidos destacamos que las principales funciones reconocidas para este alumnado son orientar $(66,15 \%)$, educar, y enseñar o transmitir conocimientos y experiencias (52,31\%), motivar $(41,54 \%)$, animar y dinamizar $(40 \%)$, informar y promover $(27,69 \%)$, facilitar procesos y tutorizar $(24,62 \%)$, evaluar $(18,46 \%)$, investigar $(16,92 \%)$, intervenir socialmente y favorecer los procesos de mediación $(13,85 \%)$ u otros aspectos como favorecer la integración social, diseñar recursos y actividades, gestionar la planificación de los procesos y observar $(12,31 \%)$.

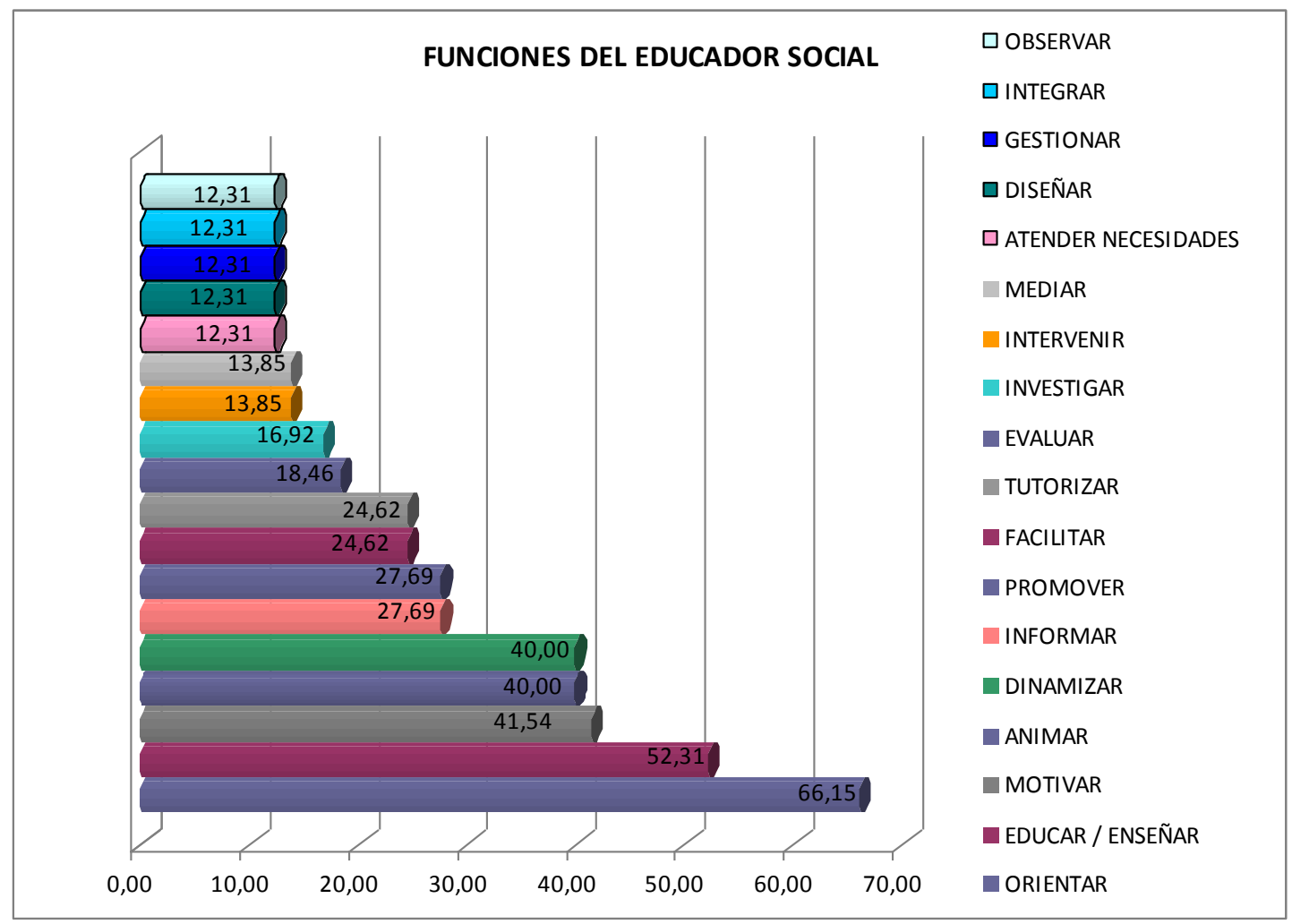

Gráfica 1. Principales funciones del educador/a social.

Siendo bastante aproximados los porcentajes en las consideraciones por sexo en la mayoría de los aspectos, aunque ellos les dan mayor importancia a las funciones de dinamizar, educar, facilitar los procesos y motivar, mientras que ellas dan mayor importancia a los aspectos relacionados con la información, la promoción y la investigación.

Estos son los conceptos más reseñados pero no los únicos, siendo reconocidas en menor porcentaje las funciones de: atender a las necesidades, comprender, evaluar, gestionar, guiar, intervenir, organizar, prevenir, conciliar, programar, concienciar, rehabilitar, ayudar, potenciar, asesorar, socializar o favorecer los procesos de autonomía. 
A continuación, y tomando las palabras utilizadas por el alumnado que ha participado en la experiencia universitaria, algunas de estas funciones quedan justificadas de la siguiente forma:

- ANIMAR "para que tengan una vida en comunidad y disfruten de lo que hacen a la vez de crecer como personas". (Paloma Romero Ruiz), "A una vida en comunidad, a una mejor socialización y a resolver conflictos a través de dinámicas y proyectos de intervención social." (Carolina Domínguez García)

- DINAMIZAR: "ejercer esta función en las actividades realizadas con diferentes colectivos al realizar actividades dinámicas". (Elena González Ruiz y Ángel Vegara Sanchez)

- DISEÑAR Y GESTIONAR ACTIVIDADES, "donde se fomente la participación grupal, atendiendo en todo momento la diversidad de ritmos" (Alba Pachón Quintero)

- EDUCAR "para enseñarles los valores necesarios para llevar una vida adecuada y tener conocimientos acerca de las cosas". (Paloma Romero Ruiz)

- FACILITAR: "Facilita al ciudadano una vida mejor en sus obstáculos" (Elena González Ruiz y Ángel Vegara Sanchez)

- INFORMAR: "Cada individuo evalúa las consecuencias posibles y adecúa sus actitudes y acciones de manera acorde a las consecuencias previsibles que se deducen del significado de la información". (Marta Hidalgo Sanz)

- INTEgRAR: "Se trata de hacer que todas las personas estén a gusto y bien socializadas con las que le rodean y con su entorno". (Carolina Domínguez García)

- INVESTIGAR: "Ya que esta sociedad está siempre en continuo cambio, de manera que el educador social tiene que estar dispuesto a cambiar e investigar para estar siempre al día". (Noelia Jimeno Juan y Cintia Caro Galavís)

- MEDIAR: "La función de mediar, que desempeña lo que está (el medio) o actúa (el mediador) en un momento intermedio de un proceso y hace posible el paso de un estado anterior a otro posterior". (Marta Hidalgo Sanz)

- MOTIVAR con el fin de "provocar de curiosidad intelectual y entusiasmo, estimulador de aprendizajes, dinamizador de los grupos de trabajo colaborativo" (Ma Carmen Solís Pérez), "el educador debe ser innovador en sus enseñanzas para de esta forma hacerlas más novedosas y atractivas a sus alumnos provocando su curiosidad intelectual" (Alba Pachón Quintero)

- ORIENTAR: Se trataría de "guía de aprendizajes y del desarrollo de las capacidades de los alumnos (debe enseñar a aprender)". (Ma Carmen Solís Pérez), "Dar pautas a quien lo necesite para que esa persona por sí sola consiga sus propios objetivos." (Carolina Domínguez García)

- PROMOVER: "Promotor del uso de las TICs, puesto que éstas son importantes para el educador social, ya que le ayudan a desempeñar su trabajo ya que, por ejemplo, existen multitud de aplicaciones en Internet gratuitas, que pueden facilitar el diálogo y la comunicación en un grupo, intercambiando ideas y experiencias. También fomentan la participación". (Noelia Jimeno Juan y Cintia Caro Galavís)

- PROVEER DE RECURSOS, "siempre ha de proporcionar los medios y recursos necesarios para llevar a cabo las actividades de forma autónoma". (Alba Pachón Quintero) 
- TUTORIZAR: "tratando de ayudar a quienes más lo necesitan, personas y colectivos en situación de marginación, desadaptación, conflicto social y exclusión social.". (Noelia Jimeno Juan y Cintia Caro Galavís)

También es de resaltar que son bastante aproximados los porcentajes en las consideraciones por sexo en la mayoría de los aspectos, aunque ellos les dan mayor importancia a las funciones de educar y facilitar los procesos, mientras que ellas dan mayor importancia a los aspectos relacionados con la orientación, la información, la promoción y la integración.

Quisiéramos reseñar que en base a las manifestaciones del alumnado, la aplicación Prezi 2.0 se constituye como plataforma de fácil manejo y aplicación para siendo un total del 31,78\% del alumnado los que hacen referencia a este aspecto y salvo una excepción que si reseña aspectos tantos positivos como negativos de la aplicación, la totalidad del alumnado la consideran positiva e innovadora. Algunos ejemplos de estas opiniones son los reflejados a continuación:

Sara Sánchez Muñoz, 1ำ de Educación Social del grupo 11.

"El trabajo me ha sorprendido ya que considero una buena forma y muy útil de realizar nuevos trabajos con otros métodos que no están tan vistos, como éste. Prezi es un fabuloso método para realizar presentaciones que tendré en cuenta para mi futuro y es lo que hemos estado trabajando en clase".

Guadalupe García Vahí, de 1o de Educación Social de la UPO.

"El Prezi, [...] Nos permite mostrar al mundo de manera muy dinámica e interactiva cualquier lección o tema [...] decir un punto negativo de estas aplicaciones, que es que debes acceder a Internet para poder usarlas, cosa que muchas veces conlleva dificultades y requiere la puesta en marcha de recursos económicos y humanos de los que a veces no disponemos". http://practica4000.jimdo.com/

En última instancia, con un $11 \%$ aparecen diferentes inconvenientes relacionados con el propio proceso de enseñanza-aprendizaje: se dice que el uso de las TIC puede favorecer un aprendizaje superficial e implicar un alto coste para la formación. También se plantea que actualmente no existen adecuados mecanismos de control en el ámbito de la teleformación.

\section{CONCLUSIONES Y LIMITACIONES DEL ESTUDIO.}

Actualmente, cada vez se cuestionan más los modelos y estrategias transmisivas de enseñanza, el aprendizaje memorístico por parte del estudiantado y su control a través de pruebas escritas. Por el contrario, se insiste en que los métodos de enseñanza deben potenciar la capacidad de aprendizaje autónomo por parte de los y las estudiantes, el desarrollo de competencias sociales, intelectuales y tecnológicas, el fomento de la reflexión colectiva y la evaluación formativa (López Meneses, Domínguez, Álvarez y Jaén, 2011). Se hace difícil pensar hoy en día en una Universidad de calidad que funcione sin el soporte de las TIC, ya que gran parte de la docencia, investigación y transferencia que ésta realiza se sustenta en las mismas (Aguaded y Hernando, 2011). En este sentido, el software social se ha convertido en un factor clave para la puesta en práctica de experiencias universitarias de innovación pedagógica en el contexto de los nuevos retos que se proponen desde el Espacio 
Europeo de Educación Superior (Aguaded y López Meneses, 2009). En este sentido, confirmamos que las nuevas tendencias tecnológicas emergentes son recursos muy valiosos para la construcción del conocimiento en los procesos de aprendizaje, propiciando la reformulación de metodologías socio-constructivistas e investigadoras. Igualmente, facilitan la gestión de la información, el desarrollo social y la innovación docente universitaria (Cabero, López Meneses y Llorente, 2009).

Entre las principales conclusiones que se obtienen, cabe mencionar la consecución de los objetivos propuestos, tanto a nivel general, quedando patente las concepciones previas que se poseen sobre la figura del Educador y el Trabajador Social, las cuales se aproximan en gran medida a aportaciones realizadas por diversos autores, que enfatizan el desarrollo de funciones en todos los ámbitos del desarrollo humano, no sólo en el educativo (Vallés, 2011; López y León, 2003; González y Revilla, 2001; López Aróstegui, 1995), como los objetivos específicos que poseen un carácter más educativo, constatándose el papel activo y autónomo desarrollado por los estudiantes en el proceso de reflexión y construcción sobre las funciones del educador/a social y trabajador/a social utilizando las presentaciones hipermedia como recurso didáctico se han conseguido plenamente, como lo demuestran los trabajos realizados por en el espacio virtual http://practica4000.jimdo.com/.

Por otra parte, como corroboran los resultados obtenidos en la práctica educativa, las presentaciones educativas multimedia son adecuadas para los procesos de aprendizaje universitario multimodal. Se ha conseguido plenamente que el estudiantado sea agente activo en su propio proceso formativo, al diseñar y elaborar de forma autónoma las presentaciones de materiales educativos interactivos, seleccionando y estructurando las principales funciones del profesional de la educación. De igual manera, se constata que la experiencia digital educativa puede facilitar el andamiaje socio-cognitivo para la construcción de un pensamiento creativo digital divergente.

Respecto a las limitaciones de la experiencia universitaria, coincidiendo parcialmente con anteriores experiencias (López Meneses y Ballesteros, 2008; Cabero, López Meneses y Ballesteros, 2009; López Meneses, E. y Llorente, M. C., 2010), indicar la falta de tiempo. También es interesante resaltar que en algunas composiciones visuales interactivas realizadas por los y las estudiantes predominaban excesivamente los textos en detrimento de lo visual y en la mayoría de los comentarios enviados al blog de la asignatura se limitaban, simplemente, a indicar algunas funciones del profesional de la educación con breves comentarios. Por otro lado, cabe mencionar la necesidad de establecer procesos de autoevaluación y heteroevaluación entre los y las estudiantes para potenciar procesos de evaluación más reflexivos y enriquecedores. En el caso concreto de nuestro estudio la falta de tiempo hizo imposible su puesta en práctica pero estamos decididos a desarrollar esta estrategia en ulteriores aproximaciones.

Por último, es adecuado que investigaciones de este tipo no se realicen de forma aislada, sino con otras Universidades, tanto nacionales o internacionales, para la creación de macrocomunidades internacionales de conocimiento compartido. En esta línea, y como otro producto de este proyecto, actualmente estamos impulsando un colectivo docente internacional interdisciplinario sobre docencia, innovación e investigación educativa, denominado Grupo Innovagogía ${ }^{\circledR}:$ http://grupoinnovagogia.blogspot.com/ 
Quisiéramos cerrar nuestra exposición resaltando que el elenco de aplicaciones relacionadas con la web 2.0 es muy amplia y puede ofrecer en el ámbito educativo nuevos espacios para la comunicación, la colaboración, la imaginación y la creación de comunidades de conocimientos compartidos. Pueden, de hecho, ser los nuevos senderos para caminar por el bosque de la innovación educativa y el desarrollo profesional del educador (Domínguez, Torres y López Meneses, 2010). Nuestra intención, dentro del marco de actuación de este proyecto de innovación docente 2.0 y en consonancia con diferentes autores (Caballo y Gradaílle, 2008; Usurriaga, 2011), es que la Educación Social mantenga activa su capacidad para promover procesos de aprendizaje, formación y desarrollo con vocación de cambio y transformación social, al menos en aquellas condiciones y circunstancias que contribuyan decisivamente al bienestar y a mejorar la calidad de vida, además de contribuir al desarrollo de las competencias culturales de comunicación de valores sociales y culturales de las personas.

Asimismo, la inclusión de las nuevas tendencias tecnológicas emergentes en las intervenciones socioeducativas es una labor indispensable para la génesis y consolidación de una sociedad informacional participativa, igualitaria e inclusiva, siempre y cuando detrás haya un profesional de la educación que, desde un enfoque ético-pedagógico, dé sentido a estos recursos didácticos para la cohesión social, el desarrollo del bienestar y la ciudadanía global (López Meneses, 2012).

\section{REFERENCIAS BIBLIOGRÁFICAS}

ACHTERMAN, D. (2006). Making connections with blogs and wikis. California School Library Association Journal, 30 (1); 29-31.

AGUADED, J. I. y LÓPEZ MENESES, E. (2009). La blogosfera educativa: nuevos espacios universitarios de innovación y formación del profesorado en el contexto europeo. Revista electrónica Interuniversitaria de formación del profesorado, 12 (3); 165172.

AGUADED, J. I., GUZMÁN, M. D. y TIRADO, R. (2010). Estudio sobre el uso e integración de Plataformas de Teleformación en universidades andaluzas. Congreso Virtual DIMAULATIC, Barcelona.

AGUADED, J. I., LÓPEZ MENESES, E y ALONSO, L. (2010a). Innovating with Blogs in University Courses: a Qualitative Study. The New Educational Review, 22 (3-4); 103-115.

AGUADED, J. I., LÓPEZ MENESES Y ALONSO, L. (2010b). Formación del profesorado y software social. Estudios sobre educación, 18; 97-114.

AGUADED, J. I. y HERNANDO, A. (2011). Recursos tecnológicos en la Universidad de Huelva: hacia la universidad digital. En CABERO, J, AGUADED, J. I.; LÓPEZ MENESES et al. Experiencias innovadoras hispano-colombianas con Tecnologías de la Información y la Comunicación. Sevilla: Mergablum, 65-85.

AIEJI. (2005). Marco Conceptual de las Competencias del Educador Social. Recuperado el 27/02/11 
AREA, M. (2009). Manual electrónico: Introducción a la Tecnología Educativa. Universidad de La Laguna. Recuperado el 21/09/11 de http://webpages.ull.es/users/manarea/ebookte.pdf

BLOOD, R. (2000). Weblogs: A History and Perspective. Recuperado el 26/09/11 de http://www.rebeccablood.net/essays/weblog history.html

BOGDAN, R. y BIKLEN, S. (1992). Investigación cualitativa de la educación. Needham Heights, MA: Allyn and Bacon.

BOHÓRQUEZ, E. (2008). El blog como recurso educativo. Recuperado el 21/09/11 de http://edutec.rediris.es/Revelec2/revelec26/edutec26 el blog como recurso educ ativo.html

CABALLO, B Y GRADAÍlLE, R. (2008). La Educación Social como práctica mediadora en la relaciones escuela-comunidad local. Revista Interuniversitaria de Pedagogía Social, 15 ; 45-55.

CABERO, J. (2003). La galaxia digital y la educación: los nuevos entornos de aprendizaje. En AGUADED, J. I. Luces en el laberinto audiovisual. Huelva: Grupo Comunicar, 102-121.

CABERO, J. (2004). Las web para la formación. En SALINAS, J.; AGUADED, J. I. y CABERO, J. (Coords.) Tecnologías para la educación. Diseño, producción y evaluación de medios para la formación docente. Madrid: Alianza, 207-229.

CABERO, J. (2005). Las TIC y las Universidades: retos, posibilidades y preocupaciones. Revista de la Educación Superior, 34 (3); 77-100.

CABERO, J.; LÓPEZ, E. y BALLESTEROS, C. (2009). Experiencias universitarias innovadoras con blogs para la mejora de la praxis educativa en el contexto europeo. Revista de Universidad y Sociedad del Conocimiento, 6. Recuperado el 28/09/11 de http://rusc.uoc.edu/ojs/index.php/rusc/article/view/v6n2 cabero etal/v6n2 cabero

CABERO, J; LÓPEZ MENESES, E. y LLORENTE, M.C. (2009). La docencia universitaria y las tecnologías web 2.0: renovación e innovación en el Espacio Europeo. Sevilla: Mergablum.

CENIH, G. y SANTOS, G. (2005). Propuesta de aprendizaje basado en proyectos y trabajo colaborativo: experiencia de un curso en línea. Revista Electrónica de Investigación $\begin{array}{lllll}\text { Educativa, } & 7 & \text { (2). } & \text { Recuperado }\end{array}$ http://redie.uabc.mx/vol7no2/contenido-cenich.html

DEL MORAL, M. E Y VILLALUSTRE, L. (2012). Didáctica universitaria en la era 2.0: competencias docentes en campus virtuales. Revista de Universidad y Sociedad del Conocimiento (RUSC), 9 (1); 36-50. Recuperado el 26/09/11 de http://rusc.uoc.edu/ojs/index.php/rusc/article/view/v9n1-moral-villalustre/v9n1moral-villalustre

DOMínGUEZ, G., TORRES, Mạ. L. y LÓPEZ MENESES, E. (2010). Aprendizaje con wikis. Usos didácticos y casos prácticos. Sevilla: MAD. 
FARMER, B., YUE, A. y BROOKS, C. (2008). Using blogging for higher order learning in large cohort university teaching: A case study. Australasian Journal of Educational Technology, 24 (2); 123-136.

GONZÁLEZ, V. y REVILLA, S. (2001). El Educador Social en Burgos. Tesis de Maestría del Master en Educación Social y Animación Sociocultural. Universidad de Granada.

GUENTHER, K. (2011). Socializing your web site with wikis, twikis, and blogs. Online, 29 (6); 51-53. Recuperado el 28/09/11 de http://www.infotoday.com/online/nov05/index.shtml

HARASIM, L. (2000). Redes de aprendizaje. Guía para la enseñanza y el aprendizaje en red. Barcelona: Gedisa.

HUFFAKER, D. (2005). The educated blogger: Using weblogs to promote literacy in the classroom. AACE Journal, 13 (2); 91-98.

LARA, T. (2005). Blogs para educar. Usos de los blogs en una pedagogía constructivista. Revista Telos. Cuadernos de Comunicación, Tecnología y Sociedad, 65; 1-15.

LÓPEZ ARÓSTEGUI, R. (1995). El perfil profesional del Educador y la Educadora Social en Euskadi. Vitoria: Gobierno Vasco.

LÓPEZ MENESES, E. y BALLESTEROS, C. (2008). Caminando hacia el software social: una experiencia universitaria con blogs. Píxel-Bit. Revista de Medios y Educación, 22; 5-23.

LÓPEZ MENESES, E y LLORENTE, Mạ. C. (2010). Incorporación de nuevas estrategias de enseñanza en la Universidad: blogs en Didáctica General. Revista Educatio Siglo XXI, 28 (1); 191-208. Recuperado el 28/09/11 de http://revistas.um.es/educatio/article/view/109781/104471

LÓPEZ MENESES, E; DOMíNGUEZ, G; ÁLVAREZ, F. J. y JAÉN, A. (2011). Experiencia didáctica con estudiantes de postgrado sobre los roles del educador en la Sociedad del Conocimiento y la Comunicación con tecnologías 2.0. Revista Latinoamericana de Tecnología Educativa, 10 (1); 49-58.

LÓPEZ MENESES, E. (2012). Educador Socia, Web 2.0 y Actitud 2.0. Madrid: Editorial Académica Española.

LÓPEZ NOGUERO, F. Y LEÓN SOLÍS, N. (2003). El educador social como educador no formal: un gestor de grupos. Ágora. Huelva. Recuperado el 26/09/11 de http://www.uhu.es/agora/version01/digital/numeros/04/04articulos/monografico/pdf 4/10.PDF

MARQUÈS, P. (2000). Los docentes: funciones, roles, competencias necesarias, formación. Recuperado el 14/09/11 de http://peremarques.pangea.org/docentes.htm

MILES, M. B. Y HUBERMAN, A. (1994). Qualitative data analysis: an expanded sourcebook. Newbury Park, CA: Sage. 
MIRANDA, M. J., GUERRA, L., FABBRI, M. Y LÓPEZ MENESES, E. (Coords.). (2010). Experiencias universitarias de innovación docente hispano-italianas en el espacio europeo de educación superior. Sevilla: Mergablum.

MONTERO, M. G. (2010). El blog como herramienta de expresión para un alumno con trastorno del espectro autista. Campo Abierto, 29 (1); 147-163.

O'DONNELL, M. (2006). Blogging as pedagogic practice: Artefact and ecology. Asia Pacific Media Educator, 17; 5-19.

PRENSKY, M. (2004). The emerging online life of the digital natives: what they do differently because of technology, and how they do it. Work in progress. Recuperado el 28/09/11 de $\quad$ http://www.marcprensky.com/writing/PrenskyThe Emerging Online Life of the Digital Native-03.pdf

PULICHINO, J. (2006). Future directions in e-Learning research report. Santa Rosa, CA, USA: The eLearning Guild.

RAY, J. (2006). Welcome to the Blogosphere: The educational use of blogs. Kappa Delta Pi Record, 42 (4); 175-177.

SAEED, N., YANG, Y. y SINNAPPAN, S. (2009). Las tecnologías web emergentes en la Educación Superior. Educational Technology \& Society, 12 (4); 98-109.

SOTO, J. (2010). TIC y profesionalización continua de docentes, los grandes horizontes del fortalecimiento educativo. Ponencia presentada en el CIVE 2010 Congreso Internacional Virtual de Educación. Recuperado el 26/09/11 de http://www.steiformacio.com/cive/

SUÁREZ, C. y LÓPEZ MENESES, E. (2011). La Universidad y los entornos educativos virtuales 2.0. En CABERO, J.; AGUADED, J. I.; LÓPEZ MENESES, E.; SANDOVAL, J. y DOMÍNGUEZ, G. Experiencias innovadoras hispano-colombianas con Tecnologías de la Información y la Comunicación. Sevilla: Mergablum, 35-45.

TELLO, J. y AGUADED, J. I. (2009). Desarrollo profesional docente ante los nuevos retos de las tecnologías de la información y la comunicación en los centros educativos. Píxel-Bit. Revista de Medios y Educación, 34; 31-47.

USURRIAGA, J. (2011). Marco conceptual de las funciones y competencias del Educador/a Social. Revista de Educación Social, 13; 1-20.

VALLÉS HERRERO, J. (2011). Análisis y valoración de las funciones de los educadores sociales en España. Tesis doctoral. Recuperado el 28/09/11 de http://espacio.uned.es/fez/eserv.php?pid=tesisuned:EducacionJvalles\&dsID=Documento.pdf

VALVERDE, J. y LÓPEZ MENESES, E. (2009). Modelos pedagógicos en la docencia universitaria a través de entornos virtuales de enseñanza-aprendizaje. Campo Abierto, 28 (2); 4768. 
HART, J. (2011). The Top 100 Tools for Learning 2011 List. Centre for Learning y Performance Technologies. (C4PLT.). Recuperado el 26/09/11 de http://c4lpt.co.uk/top-tools/top100-tools-for-learning-2011

WILLIAMS, J. B. y JACOBS, J. (2004). Exploring the use of blogs as learning spaces in the higher education sector. Australasian Journal of Educational Technology, 20 (2); 232247.

Para citar este artículo:

LÓPEZ, E.; LLORENT, V. J. \& FERNÁNDEZ, E. (2013). Experiencia universitaria sobre las funciones del educador/a social con tecnologías 2.0. EDUTEC, Revista Electrónica de Tecnología Educativa, 43. Recuperado el dd/mm/aa de http://edutec.rediris.es/Revelec2/Revelec43/experiencia universitaria funciones educador social tecnologia.html 